\title{
Characterizing Pharmacokinetic-Pharmacodynamic Relationships and Efficacy of PI3K $\delta$ Inhibitors in Respiratory Models of TH2 and TH1 Inflammation
}

\author{
Robbie L. McLeod, Malgorzata A. Gil, Dapeng Chen, Antonio Cabal, Jason Katz, \\ Joey Methot, Janice D. Woodhouse, Lauren Dorosh, Prasanthi Geda, Khamir Mehta, \\ Milenko Cicmil, Gretchen A. Baltus, Alan Bass, Hani Houshyar, Michael Caniga, \\ Hongshi Yu, Francois Gervais, Stephen Alves, and Sanjiv Shah
}

Merck \& Co., Boston, Massachusetts; Merck \& Co., Rahway, New Jersey

Received July 30, 2018; accepted February 21, 2019

\begin{abstract}
We leveraged a clinical pharmacokinetic (PK)/pharmacodynamics (PD)/efficacy relationship established with an oral phosphatidylinositol 3-kinase $(\mathrm{PI} 3 \mathrm{~K}) \delta$ inhibitor (Idelalisib) in a nasal allergen challenge study to determine whether a comparable $\mathrm{PK} / \mathrm{PD} / \mathrm{efficacy}$ relationship with $\mathrm{PI} 3 \mathrm{~K} \delta$ inhibitors was observed in preclinical respiratory models of type $2 \mathrm{~T}$ helper cell (TH2) and type $1 \mathrm{~T}$ helper cell (TH1) inflammation. Results from an in vitro rat blood basophil (CD63) activation assay were used as a PD biomarker. $\mathrm{IC}_{50}$ values for PI3K $\delta$ inhibitors, MSD-496486311, MSD-126796721, Idelalisib, and Duvelisib, were $1.2,4.8,0.8$, and $0.5 \mu \mathrm{M}$. In the ovalbumin Brown Norway $\mathrm{TH} 2$ pulmonary inflammation model, all PI3K $\delta$ inhibitors produced a dose-dependent inhibition of bronchoalveolar lavage eosinophils (maximum effect between $80 \%$ and $99 \%)$. In a follow-up experiment designed to investigate PK attributes [maximum (or peak) plasma concentration (Cmax), area under the curve (AUC), time on target (ToT)] that govern
\end{abstract}

PI3K $\delta$ efficacy, MSD-496486311 [3 mg/kg every day (QD) and $100 \mathrm{mg} / \mathrm{kg} \mathrm{QD]}$ produced $16 \%$ and $93 \%$ inhibition of eosinophils, whereas doses $(20 \mathrm{mg} / \mathrm{kg}$ QD, $10 \mathrm{mg} / \mathrm{kg}$ twice per day, and $3 \mathrm{mg} / \mathrm{kg}$ three times per day) produced $54 \%$ to $66 \%$ inhibition. Our profiling suggests that impact of PI3K $\delta$ inhibitors on eosinophils is supported by a PK target with a ToT over the course of treatment close to the PD IC 50 rather than strictly driven by AUC, Cmax, or Cmin (minimum blood plasma concentration) coverage. Additional studies in an Altenaria alternata rat model, a sheep Ascaris-sensitive sheep model, and a TH1-driven rat ozone exposure model did not challenge our hypothesis, suggesting that an $\mathrm{IC}_{50}$ level of TE (target engagement) sustained for 24 hours is required to produce efficacy in these traditional models. We conclude that the PK/PD observations in our animal models appear to align with clinical results associated with a $\mathrm{TH} 2$ airway disease.

\section{Introduction}

The use of animal models in drug discovery has historically been integral to the development of novel therapies. Unfortunately, in many disease areas, the translatability of animal data into the clinical arena has been and continues to be challenging, with notable disconnects between the apparent effectiveness of various pharmacological agents across species, including humans (McGonigle and Ruggeri, 2014; de Caestecker et al., 2015; Gidyk et al., 2015; McNamee et al., 2015). This is particularly true in heterogeneous inflammatory diseases with complex multifaceted pathophysiology such as severe asthma or chronic obstructive pulmonary disease (COPD) (Grainge et al., 2016; Han et al., 2016; Powell, 2016; Vanfleteren et al., 2016). As an example, in the early

https://doi.org/10.1124/jpet.118.252551. 1990s, a very exciting emerging area of asthma research was directed at understanding the physiology, pathology, and potential therapeutic potential relating to tachykinins, a family of neuropeptides. These include substance $P$, neurokinin $\mathrm{A}$, and neurokinin $\mathrm{B}$, which mediate inflammation, airway constriction, and cough in the lungs through their respective receptors (NK1, NK2, and NK3) (Chapman et al., 1998; Ramalho et al., 2011). Many pharmaceutical companies invested significant resources into developing tachykinergic antagonists. Unfortunately, the respiratory animal models overpredicted the actions of tachykinin antagonists compared with what was seen in patients with respiratory disease (Boot et al., 2007). With that being said, NK1 antagonists with different pharmacokinetic (PK) properties such as Aprepitant (i.e., central nervous system-penetrating capabilities) have successfully entered the human clinical market for chemotherapy-mediated nausea and vomiting (Singh et al.,

ABBREVIATIONS: APC, allophycocyanin; AUC, area under the curve; BAL, bronchoalveolar lavage; BID, twice per day; Cmax, maximum (or peak) plasma concentration; COPD, chronic obstructive pulmonary disease; FACS, fluorescence-activated cell sorter; HTRF, homogeneous timeresolved fluorescence energy transfer; IL, interleukin; PD, pharmacodynamic; PI3K, phosphatidylinositol 3-kinase; PK, pharmacokinetic; QD, every day; RL, pulmonary resistance; TH1, type $1 \mathrm{~T}$ helper cell; TH2, type $2 \mathrm{~T}$ helper cell; TID, three times per day; ToT, time on target. 
2016). As a result of pipeline attrition of new drug candidates, pharmaceutical companies are utilizing several strategies, including a better anchoring of animal to human genetics and more extensive evaluation of PK-pharmacodynamic (PD) efficacy relationships, to enhance the probability of successfully advancing novel compounds from the laboratory to human patients.

Phosphatidylinositol 3-kinases (PI3Ks) are enzymes (lipid kinases) that mainly function by phosphorylating phosphoinositides on the D3 position in the inositol ring (Fruman, 2004; Ghigo et al., 2010). Based on structure and function, there are three classes of PI3Ks, designated, I, II, and III. Class I is further partitioned into Ia and Ib subtypes (Hawkins and Stephens, 2015). PI3K $\delta$ is one of four heterodimeric class I PI3K isoforms that has engendered considerable interest as a drug target due to its central role in signaling cascading leading to initiation of inflammation responses (Ghigo et al., 2010; Barnes, 2013). The PI3K $\delta$ isoform is primarily expressed in leukocytes and utilizes lipid phosphorylation to regulate signaling pathways responsible for coordinating a broad range of cellular activities, including cell survival, proliferation, differentiation, and trafficking. That said, these drugs have been found to be well tolerated in animals and humans (Perry et al., 2018). PI3K $\delta$ also impacts tumor surveillance, and genetic deletion or pharmacologic antagonism has been demonstrated to suppress tumor growth in a broad range of solid tumors in murine syngeneic models (Stark et al., 2015). PI3K $\delta$ oncological actions may, in part, be mediated by direct regulation of immune suppressor cells, regulatory $\mathrm{T}$ cells, and myeloid-derived suppressor cells, attenuating their suppressive function, and thus enabling a more effective $\mathrm{T}$ cell-mediated antitumor response. Clinical proof of concept for selective PI3K $\delta$ inhibition has been established in several hematologic malignancies (Sanford et al., 2015; Do et al., 2016). Idelalisib (Zydelig; formerly Cal-101) recently achieved regulatory approval for treatment in follicular lymphoma and chronic lymphocytic leukemia and in combination with Rituxin in indolent non-Hodgkin lymphoma.

Pulmonary pharmacologists have been considering PI3K pathways as an anti-inflammatory target for diseases such as allergic rhinitis (Horak et la., 2016), asthma (Marwick et al., 2010; Lee et al., 2016), idiopathic pulmonary fibrosis (Yan, et al., 2014; Kral et al., 2016; Campa et al., 2018), cystic fibrosis (Marwick et al., 2010), acute lung injury (Ma et al., 2015), and COPD (Barnes, 2013; Horiguchi et al., 2015; Hsu et al., 2015). A small proof of biology study with Idelalisib in patients with allergic rhinitis, a predominately type $2 \mathrm{~T}$ helper cell (TH2) inflammatory-driven upper airway disease, was conducted by Horak et al. (2016). These investigators demonstrated that $\mathrm{PI} 3 \mathrm{~K} \delta$ inhibition with Idelalisib significantly diminished total nasal symptom scores, nasal airflow, nasal secretion weights, and congestion. Noteworthy was the employment of an ex vivo whole-blood basophil (CD63) activation assay in the study, which confirms that the PI3K pathway was effectively engaged throughout the observation/measurement period in their study. Amassing similar data in preclinical respiratory models may begin to help bridge the translatability between animal results and clinical outcomes. To this end, in the current study we describe the potency and selectivity of four PI3K $\delta$ inhibitors (MSD-496486311, MSD-126796721, Idelalisib, and Duvelisib) in in vitro enzyme and cellular assays. In addition, these compounds were profiled in in vivo studies of traditional allergic TH2 (ovalbumin-sensitized and whole-body-challenged rat; Altenaria alternata intratracheal instilled rat; Ascaris-sensitive sheep) and type $1 \mathrm{~T}$ helper cell (TH1; rat ozone whole body exposure) models, with the goal of relating CD63 potency, PK, and efficacy.

\section{Materials and Methods}

\section{Biochemical Assay}

The in vitro inhibitory potencies of MSD-496486311, MSD126796721, Idelalisib, and Duvelisib across PI3K $\alpha, \beta, \gamma$, and $\delta$ isoforms were measured by an homogeneous time-resolved fluorescence energy transfer (HTRF) assay that was developed and further optimized from a kit produced by Upstate (Millipore catalog 33-047). This procedure utilizes a preformed HTRF complex between four binding partners: 1) biotinylated Phosphatidylinositol -trisphosphate (PIP3), 2) glutathione $S$-transferase-tagged pleckstrin homology domain, 3) Europium-labeled anti-glutathione $S$-transferase monoclonal antibody, and 4) streptravidin-allophycocyanin (APC). The native PIP3 produced by PI3K activity displaces biotin-PIP3 from the pleckstrin homology domain, resulting in the dissociation of the HTRF complex and a decrease in the fluorescence signal. This loss of signal was nonlinear with respect to both increasing product and time. A correction was derived from a PIP3 standard curve run in a separate assay plate. All data were calculated using the ratio of acceptor (APC) to donor (Europium) fluorescence in each well of the assay plate. The percent inhibition for each compound concentration was calculated as follows: $\%$ inhibition $=100 \times($ fluorescence ratio $-\mathrm{CtrlB}) /(\mathrm{CtrlA}-$ CtrlB) where $\mathrm{CtrlA}=\mathrm{PI} 3 \mathrm{~K}$ reaction + known reference inhibitor

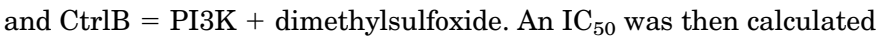
fitting the \% inhibition data to the equation: \%inhibition $=\min +$ $\left.(\operatorname{Max}-\min ) / 1+\left([\text { inhibitor }] / \mathrm{IC}_{50}\right)^{\wedge} \mathrm{n}\right)$ where min is the $\%$ inhibition with inhibitor, max was the signal in dimethylsulfoxide control, and $\mathrm{n}$ was the Hill slope.

\section{Cell-Based Assay}

The cell-based potencies of MSD-496486311, MSD-126796721, Idelalisib, and Duvelisib were measured by the ability to inhibit downstream effectors of PI3K $\delta$, as assessed by analysis of the effects of the compound on the phosphorylation status of Akt serine 473 in the Ramos lymphoma-derived B cell line. The Ramos cell line expresses cell surface IgM and responds to IgM cross-linking by activating PI3K $\delta$-dependent signaling. Treatment of Ramos cells with PI3K $\delta$ inhibitors robustly suppresses the anti-IgM-stimulated PI3K $\delta$-dependent phosphorylation of Akt S473. The cellular selectivity of PI3K $\delta$ inhibitors over other class I PI3K isoforms was determined by measuring the inhibition of Akt phosphorylation in cell-based assays selective for PI3K $\alpha$ (heregulin-stimulated SK-BR-3 cells), PI3K $\beta$ (PTEN-null MDA-MB-468 cells), or PI3K $\gamma$ (C5a-stimulated RAW 264.7 cells).

\section{In Vitro Whole-Blood Assays}

Anti-CD79b-Induced pAKT in Human Whole Blood. Human whole blood was incubated with PI3K $\delta$ inhibitors for 30 minutes and stimulated with anti-CD79B for 10 minutes at $37^{\circ} \mathrm{C}$. Samples were stained with anti-CD45, anti-CD20, and anti-pAKT; mixed; and incubated at $4^{\circ} \mathrm{C}$ for 20 minutes. Sample red blood cells were lysed for 15 minutes at room temperature and were fixed with $2 \%$ formaldehyde for 10 minutes, followed by incubation in fluorescenceactivated cell sorter (FACS) read buffer. CD20 ${ }^{+}$cells were gated.

Anti-IgE-Mediated CD63 in Rat Whole Blood. Whole blood withdrawn from male Brown Norway rats (225-275 g) was incubated with PI3K $\delta$ inhibitors for 30 minutes and stimulated with anti-IgE for 20 minutes at $37^{\circ} \mathrm{C}$. Reaction was stopped by placing at $4^{\circ} \mathrm{C}$ for 5 minutes. Samples were stained with anti-CD45-fluorescein 
isothiocyanate, anti-HAR-PE, and anti-CD63-APC into blood, mixed, and incubated at $4^{\circ} \mathrm{C}$ for 30 minutes. Sample red blood cells were lysed for 15 minutes at room temperature and were fixed with $2 \%$ formaldehyde for 10 minutes, followed by incubation in FACS read buffer. $\mathrm{CD} 45^{+} \mathrm{HARP}^{+}$cells were gated for $\mathrm{CD} 63$ analysis.

\section{Ex Vivo Whole-Blood Assay}

Anti-IgE-Mediated CD63 in Rat Whole Blood. Male Brown Norway rats were dosed with vehicle, control compound, or one of four $\mathrm{PI} 3 \mathrm{~K} \delta$ inhibitors. Whole blood was collected at day 0 to establish baseline control and then dosed at day 1 . Whole blood was then sampled at 1-, 4-, 8-, and 24-hour time points. Withdrawn blood was stimulated with anti-IgE for 20 minutes at $37^{\circ} \mathrm{C}$. Samples were stained with anti-CD45-fluorescein isothiocyanate, anti-HAR-PE, and anti-CD63-APC into blood; mixed; and incubated at $4^{\circ} \mathrm{C}$ for 30 minutes. Sample red blood cells were lysed for 15 minutes at room temperature and were fixed with $2 \%$ formaldehyde for 10 minutes, followed by incubation in FACS read buffer. CD $45^{+} \mathrm{HARP}^{+}$cells were gated for CD63 analysis.

\section{Concanavalin A-Stimulated Histamine Release in Sheep Whole Blood}

Sheep whole-blood plasma was removed, and remaining fraction was diluted with buffered saline, incubated with PI3K $\delta$ inhibitors for 30 minutes, and stimulated with $10 \mu \mathrm{g} / \mathrm{ml}$ concanavalin A for 30 minutes at $37^{\circ} \mathrm{C}$. Histamine released into supernatants was measured by enzyme-linked immunosorbent assay.

\section{Animal Care and Use}

These studies were performed in accordance with the guidelines of the Institute for Laboratory Animal Research. All studies were part of an institutional animal care and use committee-approved protocol, and animals were housed in an American Association for Accreditation of Laboratory Animal Care international accredited research facility. Because all key experimental readouts were terminal, with the exception of studies conducted in sheep, rodent study groups were used once only and were euthanized at the end of each study.

\section{Respiratory Rat Models of TH2 and TH1 Inflammation}

Ovalbumin-sensitized and challenged rat model studies were conducted by Charles River Laboratories (Montreal, QC, Canada) as per our protocol. To examine allergic-mediated lung inflammatory responses, Brown Norway rats (225-275 g) were actively sensitized to ovalbumin over a 14 -day regimen. On day 1 , animals were administered $20 \mu \mathrm{g}$ ovalbumin (i.p.) and $8 \mathrm{mg} / \mathrm{kg}$ aluminum hydroxide (i.p.) in $1 \mathrm{ml}$ physiologic saline. Seven days later, an i.p. booster containing ovalbumin $(20 \mu \mathrm{g})$ and aluminum hydroxide $(8 \mathrm{mg} / \mathrm{kg})$ was administered. Nonsensitized rats received two doses of aluminum hydroxide $(8 \mathrm{mg} / \mathrm{kg})$ administered on day 1 and day 7. Pulmonary bronchoalveolar lavage (BAL) inflammation (total and differential cell counts) was assessed following ovalbumin sensitization and challenge on day 14 using method described in detail previously (Celly et al., 2006). In the first study, we profiled the dose-response characteristics of several PIK3 $\delta$ inhibitors, MSD-496486311 (0.3-30 mg/kg), MSD126796721 (10-100 mg/kg), Idelalisib (10-100 mg/kg), Duvelisib $(0.3-30 \mathrm{mg} / \mathrm{kg})$, and the glucocorticoid agonist dexamethasone (0.003-3.0 mg/kg) for 2 days. These drugs were given orally 1 hour before ovalbumin exposure on day 14 , and BAL cellular content was examined 48 hours after ovalbumin challenge. The second experiment was designed to investigate PK attributes [maximum (or peak) plasma concentration (Cmax), area under the curve (AUC), ToT] that drive $\mathrm{PI} 3 \mathrm{~K} \delta$ efficacy in this model. We conducted a split dosing experiment, which modulated Cmax and AUC across treatment groups and allowed investigations into the PK/PD relationship. Specifically, we administered MSD-126796721 orally at $20 \mathrm{mg} / \mathrm{kg}$ every day (QD)
1 hour before ovalbumin challenge to one group of animals, then splitting the dose to $10 \mathrm{mg} / \mathrm{kg}$ twice per day (BID) and $3 \mathrm{mg} / \mathrm{kg}$ three times a day (TID) to separate groups of rats. Additionally, we tested $3 \mathrm{mg} / \mathrm{kg}$ and $100 \mathrm{mg} / \mathrm{kg}$ (QD) to round out a full dose response. Note that this dosing regimen was levied for 2 days, and BAL samples were collected 48 hours postovalbumin exposure. Separate PK studies in Brown Norway rats were conducted, as described in PK in Rats.

The rat $A$. alternata model has been described previously (Gil et al., 2014). Briefly, male Brown Norway rats (225-275 g) were purchased from Charles River Laboratories (Kingston, NY). In the first experiment, rats were intratracheally challenged with A. alternata extracts (Greer Laboratories, Lenoir, NC) in $100 \mu$ l sterile phosphate-buffered saline, using a Penn-Century microsprayer device (Penn-Century, Philadelphia, PA). MSD-126796721 (10, 30, and $100 \mathrm{mg} / \mathrm{kg}$, QD) was administered 1 hour before instillation of A. alternata. Twenty-four hours after A. alternata challenge, BAL fluid was analyzed for total inflammatory cells, neutrophils, and eosinophils population (Celly et al., 2006). The dose-response characteristics of MSD-126796721were compared with an equivalent experiment in ovalbumin-sensitized and challenged Brown Norway rat model.

Ozone studies were conducted by Pneumolabs (London, UK) as per our design. Male Brown Norway rats (200-275 g; Charles Rivers UK) were exposed to air from a primary cylinder, which passed through a Sander ozoniser (S500 MG) and into a 51 glass reservoir, where it was mixed with the air from a second inlet cylinder. The ozone output (3 ppm) was monitored using Gastec ozone detector tubes, and the generated ozone was passed through a whole-body exposure chamber $(30 \times 30 \times 45 \mathrm{~cm})$ at a rate of $2 \mathrm{l} / \mathrm{min}$. For these experiments, rats were exposed to ozone for 180 minutes, with four animals sharing a chamber at a time. Rats were given MSD-496486311 at doses of 10 (QD), 30 (QD), 15 (BID), 100 (QD), 50 (BID), and 300 (QD) $\mathrm{mg} / \mathrm{kg}$. MSD-496486311 or air was given 1 hour prior to ozone exposure. Twenty-four hours after ozone provocation, the animals were euthanized by an overdose of pentobarbital, and BAL fluid was collected. The BAL fluid collection was performed, as previously described (Celly et al., 2006). BAL fluid was analyzed for total inflammatory cells and neutrophils populations using a XT-2000iV analyzer (Sysmex).

\section{PK in Rats}

Male Brown Norway rats were dosed orally by gavage. MSD496486311 was dosed at $10,30,100$, and $300 \mathrm{mg} / \mathrm{kg}$ QD. MSD126796721 was dosed at $20 \mathrm{mg} / \mathrm{kg}$ QD. Whole blood was withdrawn immediately prior to dosing and at $0.25,0.5,1,2,4,6,8,12$, and 24 hours after drug administration. Plasma concentrations were determined using liquid chromatography-tandem mass spectrometry instruments and validated bioanalytical methods. PK parameters, including the peak plasma concentration (Cmax), the time to Cmax, and the AUC, were calculated by using Watson LIMS software (version 7.2; Thermo Electron, Philadelphia, PA). Based on those PK data, plasma concentration-time profiles resulting from BID and TID dosing at the levels used in the efficacy experiments were predicted by using Phoenix WinNonlin (Version 6.3; Pharsight, Mountain View, CA).

\section{Sheep Ascaris Model}

Sheep with a naturally occurring allergic response to Ascaris suum were used in this study. These animals have a stable and reproducible increase in pulmonary resistance (RL) following inhaled challenge with A. suum. Pulmonary resistance following compound treatment is then compared with this historical RL response to assess the effects of the compound. On the day of antigen challenge, baseline values of RL were obtained, and then three sheep were treated with a $2.0 \mathrm{mg} / \mathrm{kg}$ i.v. bolus of Idelalisib, followed by a $1.92 \mathrm{mg} / \mathrm{kg}$ infusion delivered over 9 hours (via the external jugular vein). The i.v. infusion was initiated 1 hour prior to the challenge of the sheep airways with aerosolized extracts of the antigen (A. suum extract). Plasma concentration was maintained at $\sim 4.0 \mu \mathrm{M}$ for the duration of the experiment. Measurements of RL were obtained 5-10 minutes after injection, to ensure 
TABLE 1

Biochemical potency of compounds across human class I PI3K isoforms Intrinsic potency $\mathrm{IC}_{50}$ (nanomolar).

\begin{tabular}{lcccc}
\hline Isoform & MSD-496486311 & MSD-126796721 & Idelalisib & Duvelisib \\
\hline PI3K $\delta$ & 1.3 & 5.7 & 2.2 & 0.17 \\
PI3K $\alpha$ & 190 & 525 & 1134 & 96 \\
PI3K $\beta$ & 1650 & 385 & 409 & 6 \\
PI3K $\gamma$ & 4800 & 3755 & 208 & 1.8 \\
\hline
\end{tabular}

there was no adverse effect of the drug itself on RL. Then 1 hour after the start of the infusion, the sheep were challenged with A. suum antigen. Measurements of RL were obtained immediately after challenge, hourly from 1 to 6 hours after challenge, and on the halfhour from 6.5 to 8 hours after challenge. Measurements of RL were also obtained 24 hours after challenge, followed by the 24-hour postchallenge carbachol concentration response curve to determine the postchallenge PC400. Detailed methods for this procedure can be found in the supplemental materials of Moy et al. (2013).

\section{PK/PD Modeling}

A maximum efficacy value (Emax) PK/PD model describing anti-IgE basophil degranulation CD63 expression response was established using data from in vitro and ex vivo rat whole-blood assays. For our model, $\left[\mathrm{E}=\mathrm{E} 0-\mathrm{Imax} \times \mathrm{C} /\left(\mathrm{IC}_{50}+\mathrm{C}\right)\right]$, where $\mathrm{E}$ represents $\mathrm{CD} 63$ response, $\mathrm{C}$ compound plasma concentration, E0 CD63 stimulation in the absence of compound treatment, Imax the maximum inhibitory effect, and $\mathrm{IC}_{50}$ concentration required to achieve $50 \%$ of Imax in vitro.

\section{Results}

Characterization of Novel PI3K $\delta$-Selective SmallMolecule Inhibitors in Human Biochemical Assays. We identified potent, selective inhibitors of PI3K $\delta$ during the course of our internal research efforts. Additional compounds for the described studies were selected from commercial vendors, based on publically disclosed knowledge of the target. MSD-496486311, MSD-126796721, Idelalisib, and Duvelisib selectively inhibited PI3K $\delta$ enzymatic activity (compared with $\mathrm{PI} 3 \mathrm{~K} \alpha, \mathrm{PI} 3 \mathrm{~K} \beta$, and $\mathrm{PI} 3 \mathrm{~K} \gamma$ ) with $\mathrm{IC}_{50}$ values of $1.3,5.7,2.2$, and $0.17 \mathrm{nM}$, respectively (Table 1). MSD-496486311 and MSD-126796721 were also highly selective against a broad panel of $>300$ additional kinases. For example, at $10 \mu \mathrm{M}$, MSD-496486311and MSD-126796721 did not display significant inhibition $(>50 \%)$ of any of the kinases tested (data not shown).

Characterization of Novel PI3K $\delta$-Selective SmallMolecule Inhibitors in Human Cell Assays. PI3K $\delta$ is the primary PI3K isoform that mediates B cell receptor signaling. Antigen receptor-induced AKT phosphorylation and induced CD69 expression serve as surrogate markers for PI3K $\delta$-mediated cellular activity. MSD-496486311, MSD126796721, Idelalisib, and Duvelisib inhibit B cell receptor signaling in Ramos B cells in a concentration-dependent manner with $\mathrm{IC}_{50}$ values of $20,58,6.5$, and $0.4 \mathrm{nM}$ (Table 2), respectively. These values correlated with their respective enzymatic potency (Table 1). The cellular selectivity of PI3K $\delta$ inhibitors over other PI3K isoforms was determined by measuring the inhibition of AKT phosphorylation in cellbased assays selective for $\mathrm{PI} 3 \mathrm{~K} \alpha$ (heregulin-stimulated SK-BR-3), PI3K $\beta$ (PTEN-null MDA-MB-468 cells), or PI3K $\gamma$ (C5a-stimulated RAW 264.7 cells). Consequently, MSD496486311, MSD-126796721, Idelalisib, and Duvelisib are
TABLE 2

Cell-based potency of compounds across human class I PI3K isoforms Cell-based potency $\mathrm{IC}_{50}$ (nanomolar).

\begin{tabular}{ccccc}
\hline Isoform & MSD-496486311 & MSD-126796721 & Idelalisib & Duvelisib \\
\hline PI3K $\delta^{a}$ & 20 & 58 & 6.5 & 0.4 \\
PI3K $\alpha^{b}$ & $>12,500$ & 3906 & 4755 & 3891 \\
PI3K $\beta^{c}$ & $>12,500$ & 4413 & 327 & 27 \\
PI3K $\gamma^{d}$ & 7018 & 4139 & 487 & 17 \\
\hline
\end{tabular}

${ }^{a}$ Ser473-pAKT phosphorylation in anti-IgM-stimulated Ramos lymphoma-derived $\mathrm{B}$ cells.

${ }^{b}$ Ser473-pAKT phosphorylation in heregulin-stimulated SK-BR-3 cells.

${ }^{c}$ Ser473-pAKT phosphorylation in PTEN-null MDA-MB-468 cells.

${ }^{d}$ Ser473-pAKT phosphorylation in C5a-stimulated RAW 264.7 cells.

effective and selective PI3K $\delta$ inhibitors in both enzymatic and cellular contexts and can serve as pharmacological tools to establish a PK/PD relationship in preclinical respiratory models of TH2 and TH1 inflammation.

PI3K $\delta$ Inhibitors Attenuate B Cell- and BasophilDerived PD Biomarkers in Both Rat and Human Whole Blood. The importance of monitoring relevant biomarkers to improve the efficiency of drug development is increasingly recognized. We identified and validated two PD biomarkers (CD63 in rat whole blood and anti-CD79b-induced pAKT modulation in human whole blood) in this study. MSD496486311, MSD-126796721, Idelalisib, and Duvelisib inhibited anti-CD79b-induced pAKT in human whole blood with $\mathrm{IC}_{50}$ values of $42,1534,90$, and $112 \mathrm{nM}$, respectively (Table 3), which are consistent with potencies observed in the Ramos cell-based assay. To establish a relationship between plasma exposure in preclinical models and putative translation to humans, PI3K $\delta$ inhibitors were assessed for their target coverage by evaluating basophil degranulation CD63 biomarker in rat. This very same approach was used in the clinical study by Horak et al. (2016). Anti-IgE was used to induce basophil degranulation. We then measured surface CD63 upregulation on basophils. MSD-496486311, MSD126796721, Idelalisib, and Duvelisib blocked completely in a concentration-dependent manner with $\mathrm{IC}_{50}$ values (ex vivo) of $300,1200,490$, and $96 \mathrm{nM}$, respectively (Table 3).The in vitro $\mathrm{IC}_{50}$ values are also shown in Table 3.

PK/PD Modeling. A direct relationship was observed when plasma concentrations were correlated to the PD effect. These time-course and dose-response data and the estimated PK/PD parameters led to the establishment of an Emax mathematical model linking plasma concentrations to CD63 basophil degranulation inhibition over time, in which a correlation factor was calculated between predicted versus experimentally measured CD63 inhibition. Thus, a quantitative relationship between dosing regimen, exposure, and PD effects of PI3K $\delta$ was identified based on the correlation of ex vivo $\mathrm{PD}$ effects potency, which allowed for the extrapolation of a fitting mathematical PK/PD model.

Respiratory Rat Models of TH2 and TH1 Inflammation. In sensitized rats, ovalbumin exposure on day 14 produced a consistent increase in the number of BAL eosinophils ranging from $124 \pm 15$ to $160 \pm 21 \times 10^{3}$ cells $/ \mathrm{ml}$. All PIK3 $\delta$ inhibitors tested inhibited allergicinduced eosinophilic inflammation (Fig. 1). The maximum achievable efficacy of the PI3K $\delta$ inhibitors was similar to the positive comparator dexamethasone $(3 \mathrm{mg} / \mathrm{kg})$. Efficacy and simulated PK results from the MSD-126796721 split dosing study are displayed in Fig. 2 . The $3 \mathrm{mg} / \mathrm{kg} \mathrm{QD}$ and the 
TABLE 3

Potency of compounds in human or rat whole blood

Whole-blood potency $\mathrm{IC}_{50}$ (nanomolar).

\begin{tabular}{lcccc}
\hline \multicolumn{1}{c}{ Biomarker } & MSD-496486311 & MSD-126796721 & Idelalisib & Duvelisib \\
\hline Human pAKT (in vitro) & 42 & 1534 & 90 & 112 \\
Rat CD63 (in vitro) & 1200 & 4800 & 800 & 500 \\
Rat CD63 (ex vivo) & 300 & 1200 & 490 & 96 \\
\hline
\end{tabular}

$100 \mathrm{mg} / \mathrm{kg}$ QD dose levels produced $16 \%$ and $93 \%$ inhibition of BAL eosinophils, respectively, whereas the $20 \mathrm{mg} / \mathrm{kg}$ QD, $10 \mathrm{mg} / \mathrm{kg}$ BID, and the $3 \mathrm{mg} / \mathrm{kg}$ TID doses produced equivalent effects ranging from $54 \%$ to $66 \%$ BAL inflammation (Fig. 2; Table 4). Figure 2 also displays the effect of PI3K $\delta$ inhibition on TH2 BAL cytokine secretion, which mimicked these drug actions on BAL inflammation. The data shown in Table 4 support that the PK target is consistent with time on target (ToT) rather than strictly Cmax, or Cmin (minimum blood plasma concentration) driven. Modeling data relating time above the in vitro CD63 IC $_{50}$ assay and in vivo efficacy of the PI3K inhibitors support the ToT as being an important driver of efficacy in the ovalbumin-sensitized and challenged model (Fig. 3).

MSD-126796721 (10-100 mg/kg) produced a dosedependent attenuation of the increases in eosinophils produced by intratracheal instillation of A. alternata (Fig. 4). In the allergic rat models, our aim was to determine the impact of PI3K $\delta$ inhibition on TH2 inflammation. Thus, we focused our attention on eosinophils as a primary endpoint. However, it is noteworthy to point out that PI3K $\delta$ efficacy was observed on total cell counts and other BAL immune cell types (data not shown). Similarly, MSD-126796721 (10-100 mg/kg) produced dose-related inhibition of an eosinophilic pulmonary response in the BAL of Brown Norway rats sensitized and challenged with ovalbumin. Although the degree of eosinophilic inflammation was greater in the A. alternata phenotype compared with the ovalbumin model, the normalized (percent inhibition) BAL cellular response produced by PI3K $\delta$ inhibition was comparable across the two studies (Figs. 2 and 4). Additionally, the terminal plasma exposure of MSD-126796721 (10, 30, and $100 \mathrm{mg} / \mathrm{kg}$ ) was equivalent across the A. alternata $(0.052$, 0.155 and $0.428 \mu \mathrm{M})$ and ovalbumin $(0.066,0.259$, and $0.600 \mu \mathrm{M})$ models.
A

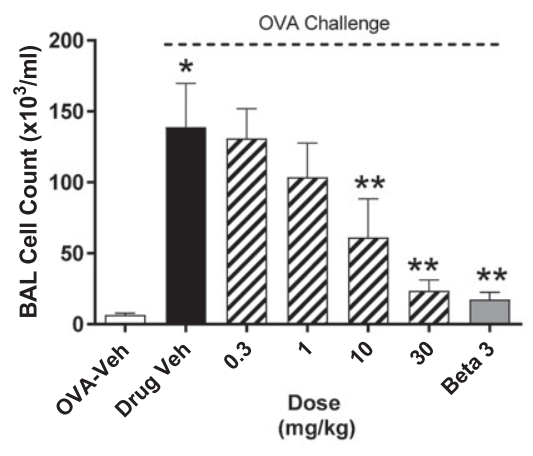

B

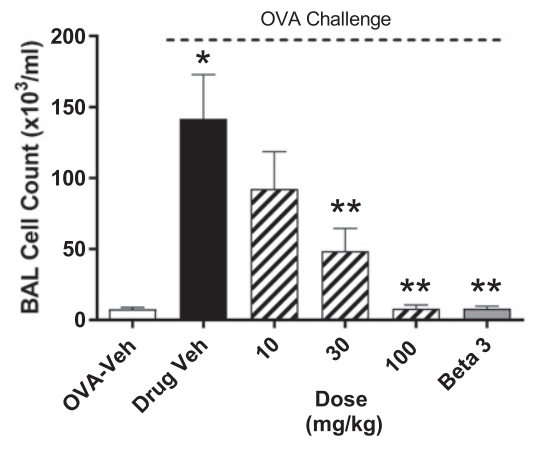

C

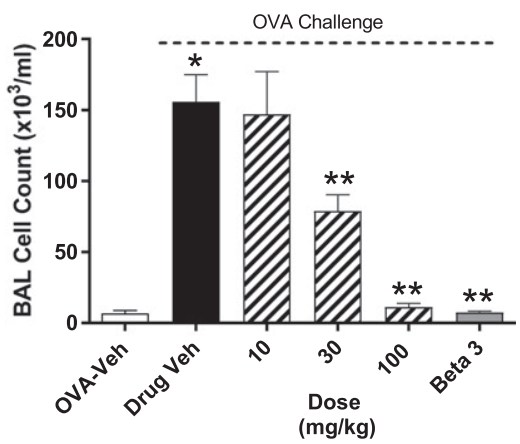

D

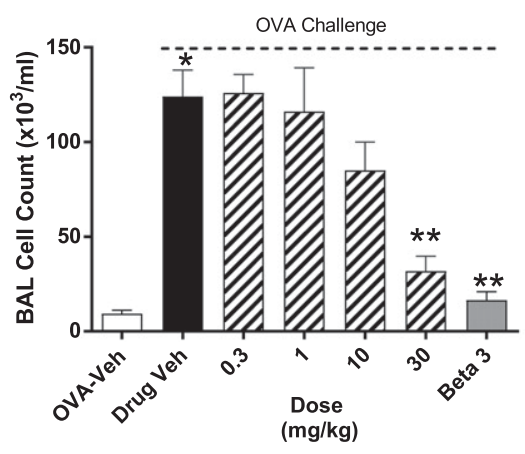

E

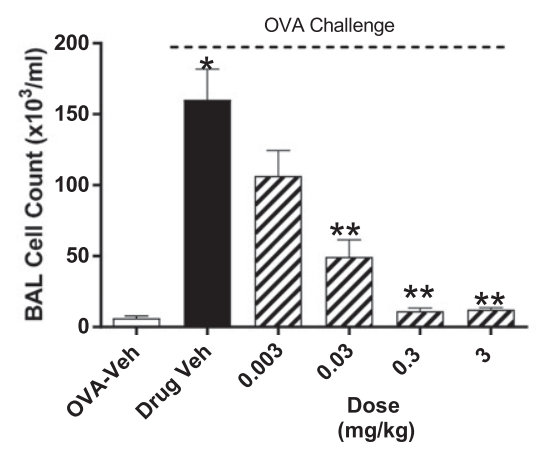

Fig. 1. PI3K $\delta$ inhibition attenuates allergic-mediated pulmonary inflammation in the ovalbumin-sensitized and challenged Brown Norway rat. Displayed are the actions of MSD-496486311 (A), MSD-126796721 (B), Idelalisib (C), and Duvelisib (D) on eosinophils 48 hours postantigen challenge. Each bar represents the mean \pm S.E.M. $(n=8$ per treatment group). $* P<0.05$ compared with vehicle control; $* * P<0.05$ vs. OVA control group. As a comparator, the effects of dexamethasone on antigen-mediated inflammation are shown in (E). 


\section{A PK Profile of} MSD-12679672

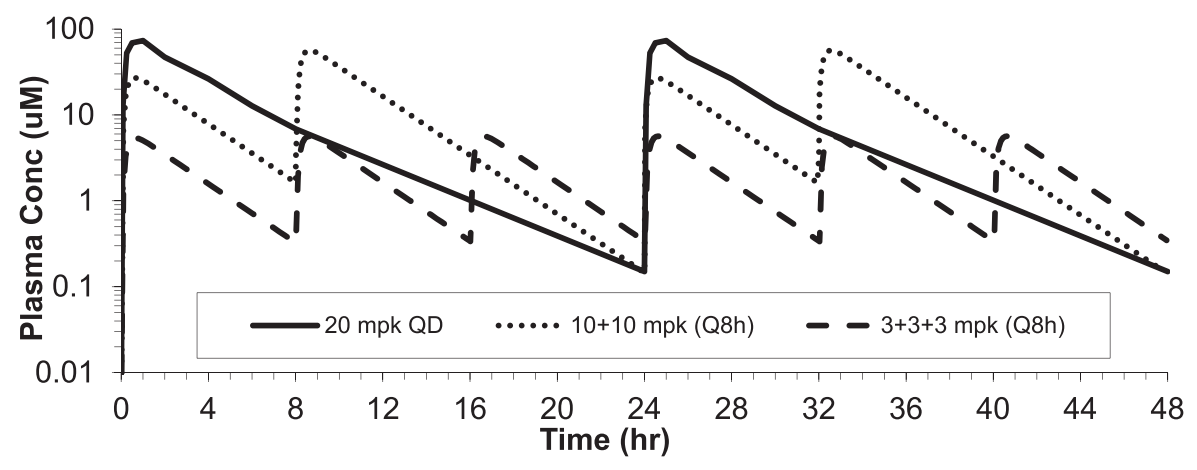

B

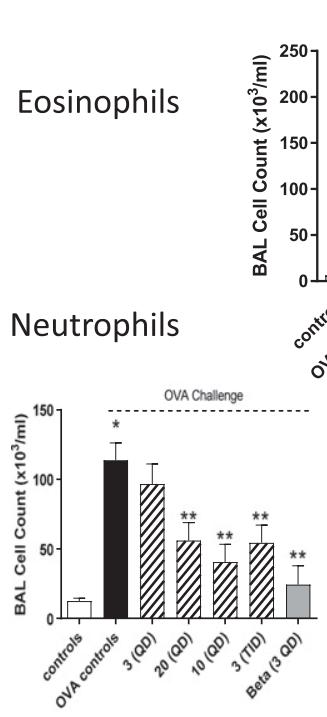

Inflammatory Cells (MSD-126796721)

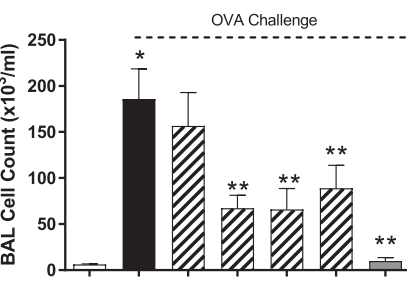

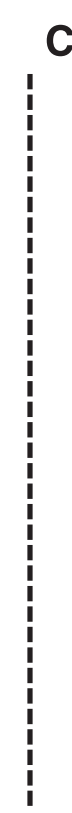

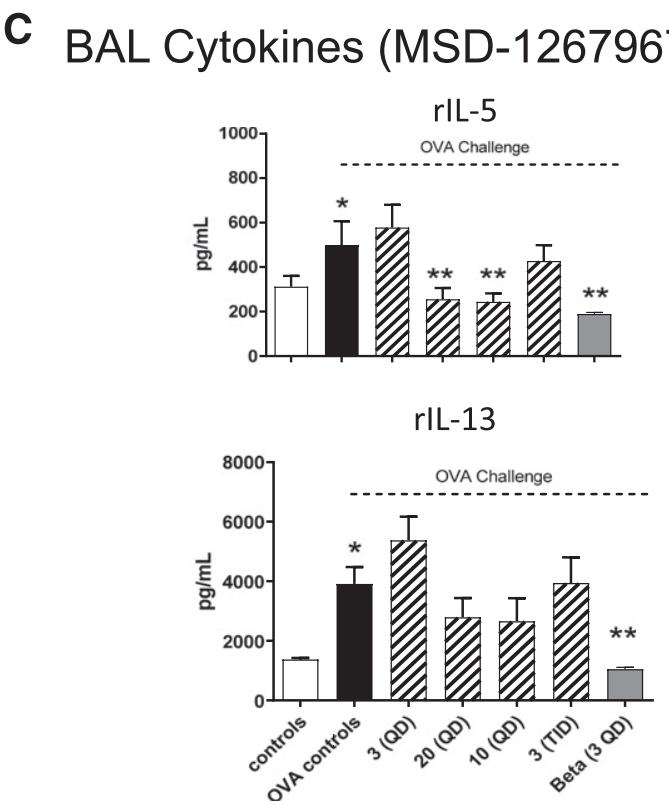

Fig. 2. Efficacy of PI3K $\delta$ inhibition in a split dosing paradigm. (A) Displays the simulated plasma exposures of MSD-126796721 after different dosing paradigms based on a two-compartment model relative to the drug's in vitro $\mathrm{IC}_{50}$ basophil CD63 value. Shown in (B and C) are the inhibitory actions of MSD-126796721 (3 mg/kg QD, $20 \mathrm{mg} / \mathrm{kg}$ QD, $10 \mathrm{mg} / \mathrm{kg}$ QD, and $3 \mathrm{mg} / \mathrm{kg}$ TID) on inflammatory TH2 BAL cytokines. Each bar represents the mean \pm S.E. M. ( $n=8$ per treatment group). $* P<0.05$ compared with vehicle control; $* * P<0.05$ vs. ovalbumin (OVA) control group. As a positive comparator, the effects of dexamethasone ( $3 \mathrm{mg} / \mathrm{kg} \mathrm{QD})$ are also displayed in the figure.

Similar to studies conducted in the ovalbumin-sensitized and challenged model, we performed a split dosing experiment in the rat ozone model. Vehicle control animals challenged with ozone displayed a significant increase in the number of neutrophils and total cells in the BAL (Fig. 5). MSD$496486311(10,30,100$, and $300 \mathrm{mg} / \mathrm{kg}$, QD, by mouth) elicited a dose-related attenuation in the ozone pulmonary inflammatory response. The split doses (i.e., $15 \mathrm{mg} / \mathrm{kg}$ BID and $50 \mathrm{mg} / \mathrm{kg}$ BID) produced comparable effects on BAL neutrophils and total cells. The PK profile of MSD-496486311 is shown in Fig. 5 and Table 5. Results in Table 5 indicate that the efficacy in this model is not driven by Cmax or AUC, but is more likely driven by $\mathrm{To} T$ above the in vitro $\mathrm{CD} 63 \mathrm{IC}_{50}$ value.

Ascaris-Sensitive Sheep Model. We evaluated the ability of clinical benchmark PI3K $\delta$ inhibitor Idelalisib to attenuate airway resistance induced by aerosolized ascaris to the lungs. Idelalisib was dosed by i.v. infusion and achieved a $5 \mu \mathrm{M}$ steady state for study duration (i.e., 9 hours). This level

TABLE 4

MSD-126796721 split dosing experiment in the Brown Norway rat OVA allergic lung inflammation model

The data are indicative of a time on target PK target. Cmax and AUC values from BID and TID studies were predicted from the simulation using Phoenix WinNonlin software. Cmax and AUC values from 3, 20, to $100 \mathrm{mg} / \mathrm{kg}$ QD studies were measured from a separate PK study.

\begin{tabular}{|c|c|c|c|c|c|c|c|}
\hline $\begin{array}{c}\text { Dose } \\
(\mathrm{mg} / \mathrm{kg})\end{array}$ & $\begin{array}{l}\mathrm{C}_{\max } \\
(\mu \mathrm{M})\end{array}$ & $\operatorname{AUC}_{0-48}(\mu \mathrm{M}$ & $\begin{array}{c}\mathrm{C}_{\text {trough }} \\
(\mu \mathrm{M})\end{array}$ & ToT $\left(>\operatorname{rat} \underset{(\mathrm{h})}{\mathrm{CD} 63 \mathrm{IC}_{50}} 48 \mathrm{~h}\right)$ & $\begin{array}{l}\text { BAL Eosinophils } \\
\text { (\% Inhibition) }\end{array}$ & $\begin{array}{l}\text { BAL Neutrophils } \\
\text { (\% Inhibition) }\end{array}$ & $\begin{array}{c}\text { BAL Totals } \\
\text { (\% Inhibition) }\end{array}$ \\
\hline 10 (BID) & 20 & 230 & 0.34 & 32 & 67 & 72 & 84 \\
\hline 3 (TID) & 6 & 103 & 0.4 & 26 & 54 & 59 & 59 \\
\hline $100(\mathrm{QD})$ & 192 & 115 & 0.9 & 42 & 93 & 96 & $>100$ \\
\hline
\end{tabular}


A Eosinophil Inhibition (\%)

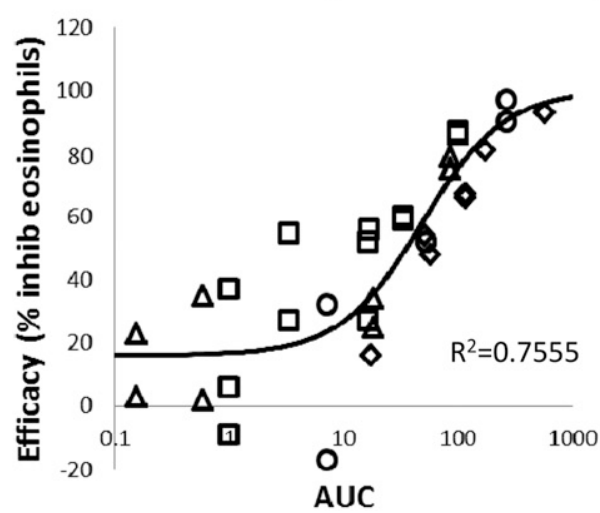

B

○ MSD-126796721

ㅁ MSD-496486311

- Idelalisib

$\Delta$ Duvelisib

Fig. 3. Correlation graph: Efficacy of pulmonary eosinophil influx to AUC (A) and efficacy of pulmonary eosinophil to ToT (B).

of exposure represented an approximate 2.5-fold over the in vitro sheep washed whole-blood basophil histamine $\mathrm{IC}_{50}$ value of $0.638 \mu \mathrm{M}(2.0 \mu \mathrm{M}$ adjusted for sheep PPB [plasma protein binding]). At the single dose tested, Idelalisib significantly attenuated early and late airway hyper-responsiveness by $40 \% \pm 5 \%$ and $78 \% \pm 8 \%$, respectively (Fig. 6 ). The airway hyper-responsiveness was fully blocked by. Idelalisib.

\section{Discussion}

Respiratory diseases such as asthma and COPD continue to be associated with substantial morbidity and mortality with a significant portion of patients being poorly controlled by current standard of care (Grainge et al., 2016; Han et al., 2016; Powell, 2016). Novel treatments, particularly for COPD, have been difficult to advance to the market place (Barnes, 2013), in part because promising pharmacological results from animal studies have not always translated into effective human efficacy (Wendler and Wehling, 2010). A better understanding of target engagement in historical models, and whenever possible linking those results to PK/PD observations in the clinic, may help offset drug pipeline failures (Danhof et al., 2008). In the current study, we leveraged published clinical data from Horak et al. (2016), which indicated Idelalisib (a selective $\mathrm{PI} 3 \mathrm{~K} \delta$ inhibitor) provided meaningful benefit to allergic rhinitis patients in a 7-day grass pollen Vienna Challenge Chamber crossover study. In addition to efficacy endpoints, the study employed an ex vivo whole-blood basophil activation assay as a surrogate PI3K $\delta$ target engagement PD marker. An Idelalisib dose level of $100 \mathrm{mg} / \mathrm{kg}$ BID produced a plasma exposure concentration of $792.7 \mathrm{ng} / \mathrm{ml}$ (3 hours after the first dose), which corresponded to a $59 \%$ (value minus control responses) suppression of ex vivo basophil activation. Consequently, we sought to understand the relationship between attenuation of whole-blood basophil activation, plasma exposure, and respiratory anti-inflammatory effects of PI3K $\delta$ inhibitors in preclinical pulmonary animal models.

Evaluation of PI3K $\delta$ inhibitors in human whole blood enables human dose projections. The panel of PI3K $\delta$ inhibitors was tested systematically across individual human donors for their ability to inhibit basophil activation in response to antiIgE. In addition, PI3K $\delta$ inhibitors were found to display similar potencies in an alternative human whole-blood assay measuring signaling via measurement of pAKT. Collectively, application of these PD biomarkers across species not only
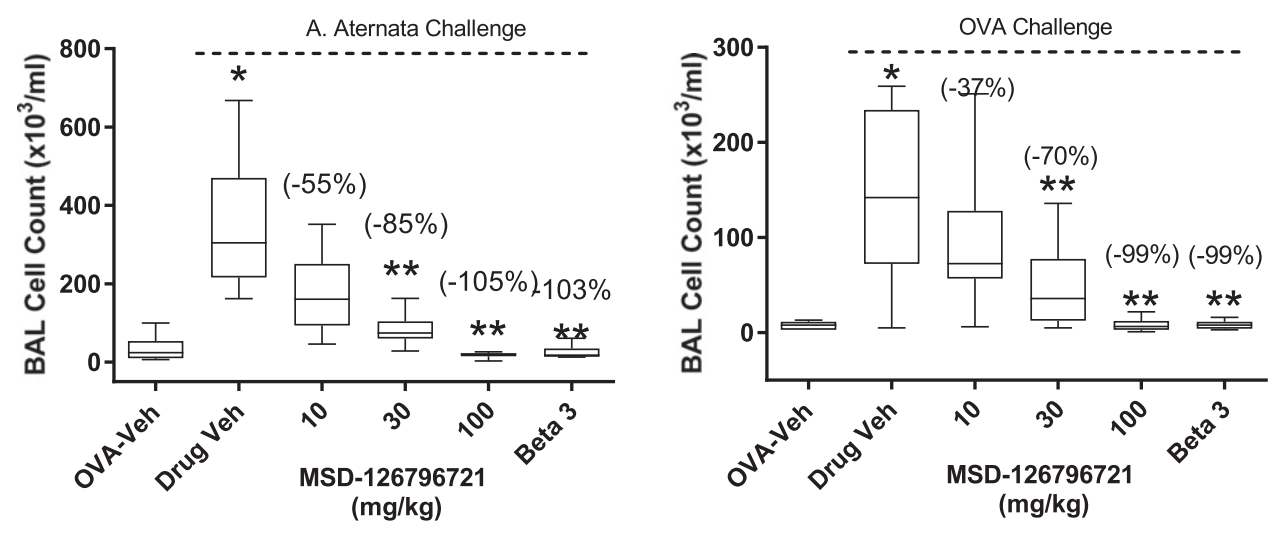

Fig. 4. Comparative anti-inflammatory effect of PI3K $\delta$ inhibition in the ovalbuminsensitized challenged and (A) alternate Brown Norway rat models. The dose-response inhibitory actions of MSD-126796721 on allergic-mediated pulmonary eosinophilic responses 48 hours postantigen challenge in the ovalbumin-sensitized challenged (A) and the A. alternata (B) models are depicted. Each bar represents the mean \pm S.E.M. $(n=$ 8 per treatment group). $* P<0.05$ compared with vehicle control; $* * P<0.05$ vs. ovalbumin (OVA) control or A. alternata group. Values in parentheses are mean percent change values from the challenged response in the control group. 

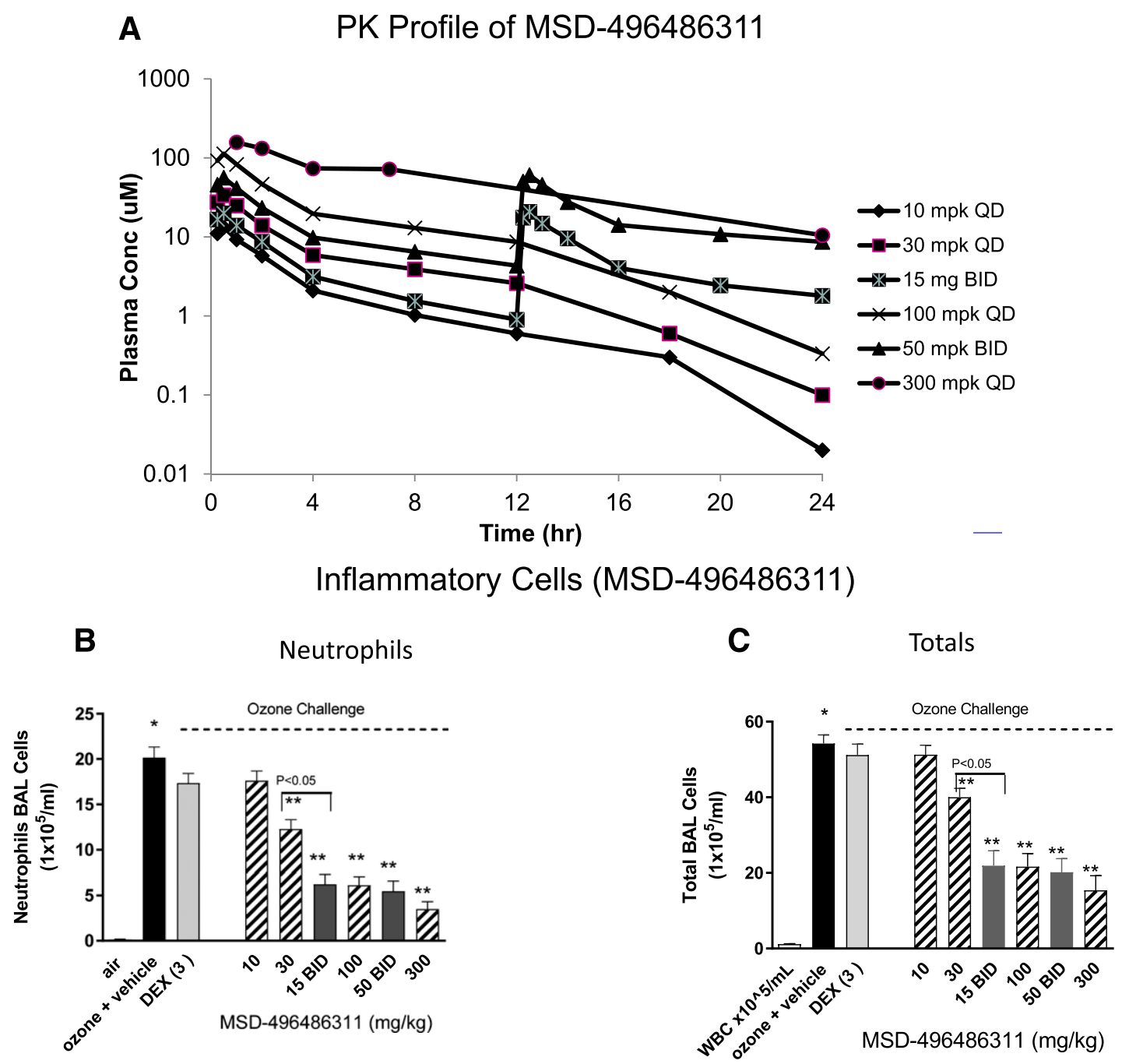

Fig. 5. Efficacy of PI3K $\delta$ inhibition in a split dosing paradigm. (A) Displays the simulated plasma exposures of MSD-496486311 after different dosing paradigms based on a two-compartment model relative to the drug's in vitro $\mathrm{IC}_{50}$ basophil CD63 value. Shown in (B and C) are the inhibitory actions of MSD-496486311 (10 mg/kg QD, $30 \mathrm{mg} / \mathrm{kg}$ QD, $15 \mathrm{mg} / \mathrm{kg}$ BID, $100 \mathrm{mg} / \mathrm{kg}$ QD, $50 \mathrm{mg} / \mathrm{kg}$ BID, and $300 \mathrm{mg} / \mathrm{kg}$ QD) on TH1-driven BAL inflammation. Each bar represents the mean \pm S.E.M. $\left(n=8\right.$ per treatment group). ${ }^{*} P<0.05$ compared with vehicle control; $* * P<0.05$ vs. ovalbumin control group.

validated their biologic relevance, but allowed us to determine the exposure-effect relationship and the correlation between $\mathrm{PI} 3 \mathrm{~K} \delta$ inhibition and efficacy in preclinical respiratory models of TH2 and TH1 inflammation.

In the current in vivo experiments, we both recapitulate and expand upon literature reports indicating that PI3K $\delta$ inhibitors can effectively attenuate both TH2- and TH1-evoked inflammatory responses in the lungs (Chen et al., 2004; Finan and Thomas, 2004; Doukas et al., 2009; Takeda et al., 2010). Our rodent studies were conducted in the Brown Norway rat, as this strain is genetically TH2-predisponsed and produces robust and consistent pulmonary inflammatory responses after allergic sensitization and challenge (Hylkema et al., 2002). Moreover, TH1 respiratory inflammatory responses can also be evoked in Brown Norway rats by various stimuli, such as ozone, lipopolysaccharide, and cigarette smoke

TABLE 5

MSD-496486311 split dosing experiment in the ozone inflammation model

The data are indicative of a time on target PK target. Cmax and AUC values from BID and TID studies were predicted from the simulation using Phoenix WinNonlin software. Cmax and AUC values from $30 \mathrm{mg} / \mathrm{kg}$ QD studies were measured from a separate PK study.

\begin{tabular}{lcccc}
\hline Dose $(\mathrm{mg} / \mathrm{kg})$ & $\mathrm{ToT}\left(0.3 \mu \mathrm{M} \mathrm{IC}_{50}\right)(\mathrm{h})$ & $\mathrm{AUC}(\mu \mathrm{M} \bullet \mathrm{h})$ & $\mathrm{Cmax}(\mu \mathrm{M})$ & Efficacy Neutrophils (\% Inhibition) \\
\hline $10(\mathrm{QD})$ & 18 & 37 & 13 & 12 \\
30 (QD) & 18 & 106 & 34 & 38 \\
15 (BID) & 24 & 114 & 21 & 69 \\
100 (QD) & 24 & 353 & 113 & 73 \\
50 (BID) & 24 & 372 & 56 & 84 \\
300 (QD) & 24 & 1270 & 158 & \\
\hline
\end{tabular}



A

\section{Lung Function}

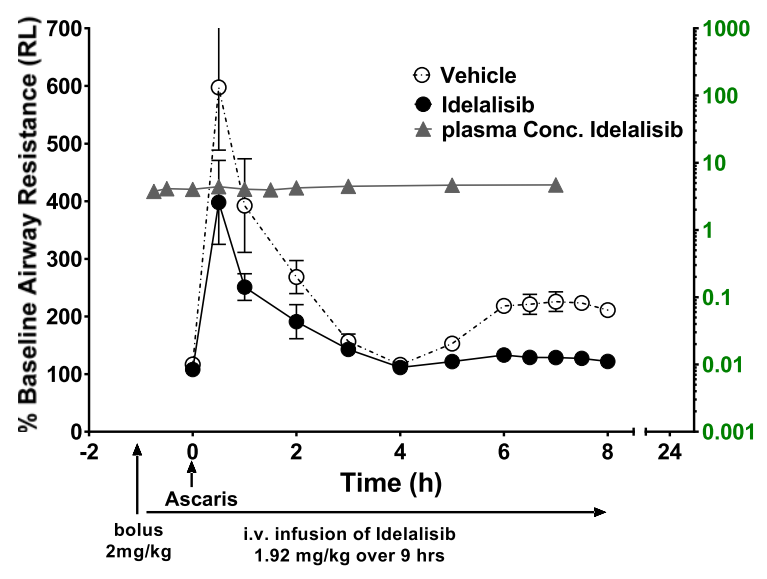

B

Airway

hyperresponsiveness

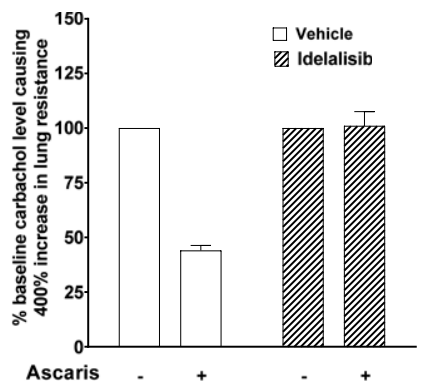

Fig. 6. Idelalisib (steady state i.v. administration@ @ $5 \mu \mathrm{M}$ ) improved lung function in the allergic sheep model. The upper insert demonstrates that Idelalisib reversed carbachol airway hyper-responsiveness Panel B. The lower insert depicts the PK profile relative to in vitro wash blood basophil $\mathrm{IC}_{50}$ (Panel A). Each point/bar represents the mean \pm S.E.M. $(n=3$ sheep per treatment group). A statistically significant $(P<0.05)$ effect on early airway hyper-responsiveness, late airway hyper-responsiveness, and airway hyper-responsiveness compared with vehicle control was observed.
(Tsukagoshi et la., 1995; Hisada et al., 1999; Bassett et al., 2000). Using the same strain eliminates confounding interpretation of our results as we associate efficacy to PK and to the in vitro basophil activation levels. Based on the clinical observations by Horak et al. (2016), we speculated that maintaining PI3K $\delta$ target engagement or ToT at our basophil CD63 IC $_{50}$ value would elicit significant efficacy in the models tested. To evaluate the effectiveness of a ToT above a threshold concentration set by the $\mathrm{CD} 63 \mathrm{IC}_{50}$, we conducted a split dosing study in the Brown Norway rat ovalbumin lung allergen challenge model with MSD-126796721 at $20 \mathrm{mg} / \mathrm{kg}$ $\mathrm{QD}, 10 \mathrm{mg} / \mathrm{kg}$ BID, and $3 \mathrm{mg} / \mathrm{kg}$ TID, which modulated Cmax and AUC across these treatment groups. This study allowed investigations into the exposure-response relationship, and the concentration-time profiles were used to compute AUC, extract other PK parameters (Cmax, ToT, Cmin), and assess their relationships with percent inhibition of eosinophils. An Emax model, as illustrated in Fig. 4A, provides a good representation of the AUC-PD relationship with multiple PI3K $\delta$ inhibitors. The use of Emax models is limited to cases where the dose range covers the entire spectrum of the response; otherwise, they are unreliable to make predictions outside the linear portion of the curve. Indeed, our initial exploration of the PK-PD relationship showed that AUC provides reasonable exposure response projections through an Emax model. Evaluation of the PD response against other PK parameters revealed that ToT provides the most robust estimate. It provides a simple linear relationship that correlates well (see Fig. 4B) with the response and does not have the stringent dose-range requirements imposed by the Emax model. Recognizing that AUC and ToT are not completely independent parameters, our data strongly support previous findings regarding the use of ToT to make reasonable dose-response projections. The impact of PI3K $\delta$ inhibition on BAL TH2 cytokines such as interleukin (IL)-5 and IL-13 paralleled the effects on BAL cells in the Brown Norway rat ovalbumin model. Interesting, at the equal doses, PI3K $\delta$ inhibition (MSD-126796721) in the A. alternata model (on a Brown Norway strain) displayed a qualitatively equivalent anti-inflammatory action to those in the ovalbumin model, suggesting a similar PK/PD relationship. Additional support for the ToT hypothesis is suggested in a second species by a small allergic sheep study, where i.v. infusion of compound C, at 2.5-fold above an in vitro whole-blood basophil histamine $\mathrm{IC}_{50}$ value, attenuated early and late airway hyperresponsiveness. Nonetheless, additional sheep experiments would be needed to fully support this position. Taken together, the data in our allergic models are in alignment with the allergic rhinitis $\mathrm{PI} 3 \mathrm{~K} \delta$ inhibitor proof of biology study by Horak et al. (2016) in regard to the degree of target engagement needed to produce respiratory anti-inflammatory responses, which is not surprising, given that allergic rhinitis and allergic lung allergen challenge preclinical models share a common pathophysiology, (i.e., Th2-type inflammation).

Th2 cytokines, including IL-4, IL-5, IL-9, and IL-13, have long been recognized as having a key function in the pathogenesis of atopic asthma and IgE-mediated eosinophilic inflammation of the lungs (Romagnani, 2002; Fishcer et al., 2007). Moreover, Th1 pathway mediators, including IL-1b, IL-8 and IL-17, and lung neutrophilia are also believed to play a key role in certain severe asthmatic populations (Wenzel et al., 1999; Douwes et al., 2002), as well as contributor to the underlying inflammation in COPD (Caramori et al., 2014; Barnes, 2015). In the current experiments, we used wholebody ozone exposure to increase total cell and neutrophil influx into the BAL of Brown Norway rats. We conducted a split dose experiment similar to the experiment design mentioned above for the ovalbumin model and observed that 
24-hour coverage of MSD-496486311 (duration to the ozone experiment) at the basophil $\mathrm{IC}_{50}$ value resulted in maximum BAL anti-inflammatory efficacy between $69 \%$ and $84 \%$ (Table 4). In contrast, the relationships between AUC, Cmax, and efficacy were not as clear in the ozone experiment.

Combination treatments such as Trelegy Ellipta, Combivent, Advair, Symbicort, and Dulera are becoming more common place for the treatment of respiratory diseases (Calzetta et al., 2019). For example, administration of triple therapy by adding a LAMA (long acting bronchodilator) to ICS (inhaled corticosteroid)/LABA (long acting bronchodilator) combination provides significant clinical benefit to asthmatic and COPD patients over ICS/LABA alone. Although combination treatments offer the possibility of improving a desired efficacy, it must be pointed out that the emergence of greater unwanted side effects may become more evident. A good understanding of PK/PD relationships may offset overdosing and minimize the liability of combination treatments. Although we did not currently study the impact of coadministration of $\mathrm{PI} 3 \mathrm{~K} \delta$ inhibitors with other respiratory drugs, we believe that target engagement experiments (such as presented in this work) can be the basis of future explorations. From our point of view, this can be expanded to potential novel respiratory targets such as NOX4 and Nrf2 (Zhao et al., 2015; Zhou et al., 2016; Cui et al., 2018).

In summary, we studied the anti-inflammatory actions of four PI3K $\delta$ inhibitors in animal models of Th1 and Th2 inflammation. We conclude that the PK/PD observations in our animal models appear to align with clinical results associated with a Th2 airway disease, allergic rhinitis.

\section{Authorship Contributions}

Participated in research design: McLeod, Shah, Cabal, Cicmil, Gervais, Bass, Alves, Houshyar.

Conducted experiments: Gil, Chen, Woodhouse, Dorosh, Geda, Mehta, Baltus, Caniga, Yu.

Contributed new reagents or analytic tools: Katz, Methot.

Performed data analysis: Yu, Shah, McLeod, Gil, Chen, Cabal.

Wrote or contributed to the writing of the manuscript: McLeod, Shah, Cabal, Caniga.

\section{References}

Barnes PJ (2013) New anti-inflammatory targets for chronic obstructive pulmonary disease. Nat Rev Drug Discov 12:543-559.

Barnes PJ (2015) Therapeutic approaches to asthma-chronic obstructive pulmonary disease overlap syndromes. J Allergy Clin Immunol 136:531-545.

Bassett DJ, Elbon-Copp C, Ishii Y, Barraclough-Mitchell H, and Yang H (2000) Lung tissue neutrophil content as a determinant of ozone-induced injury. J Toxicol Environ Health A 60:513-530.

Boot JD, de Haas S, Tarasevych S, Roy C, Wang L, Amin D, Cohen J, Sterk PJ, Miller B, Paccaly A, et al. (2007) Effect of an NK1/NK2 receptor antagonist on airway responses and inflammation to allergen in asthma. Am J Respir Crit Care Med 175: $450-457$.

Calzetta L, Cazzola M, Matera MG, and Rogliani P (2019) Adding a LAMA to ICS/ LABA therapy: a meta-analysis of triple combination therapy in COPD. Chest DOI: 10.1016/j.chest.2018.12.016 [published ahead of print].

Campa CC, Silva RL, Margaria JP, Pirali T, Mattos MS, Kraemer LR, Reis DC, Grosa G, Copperi F, Dalmarco EM, et al. (2018) Inhalation of the prodrug PI3K inhibitor CL27c improves lung function in asthma and fibrosis. Nat Commun 9:5232.

Caramori G, Adcock IM, Di Stefano A, and Chung KF (2014) Cytokine inhibition in the treatment of COPD. Int J Chron Obstruct Pulmon Dis 9:397-412.

Celly CS, House A, Sehring SJ, Zhang XY, Jones H, Hey JA, Egan RW, and Chapman RW (2006) Temporal profile of forced expiratory lung function in allergenchallenged Brown-Norway rats. Eur $J$ Pharmacol 540:147-154.

Chapman RW, Sehring SJ, Garlisi CG, Falcone A, Kung TT, Stelts D, Minnicozzi M, Jones H, Umland S, Egan RW, et al. (1998) Anti-inflammatory activity of inhaled mometasone furoate in allergic mice. Arzneimittelforschung 48:384-391.

Chen L, Martinez O, Overbergh L, Mathieu C, Prabhakar BS, and Chan LS (2004) Early up-regulation of Th2 cytokines and late surge of Th1 cytokines in an atopic dermatitis model. Clin Exp Immunol 138:375-387.

Cui W, Zhang Z, Zhang P, Qu J, Zheng C, Mo X, Zhou W, Xu L, Yao H, and Gao J (2018) Nrf2 attenuates inflammatory response in COPD/emphysema: crosstalk with Wnt3a/ $\beta$-catenin and AMPK pathways. J Cell Mol Med 22:3514-3525.
Danhof M, de Lange EC, Della Pasqua OE, Ploeger BA, and Voskuyl RA (2008) Mechanism-based pharmacokinetic-pharmacodynamic (PK-PD) modeling in translational drug research. Trends Pharmacol Sci 29:186-191.

de Caestecker M, Humphreys BD, Liu KD, Fissell WH, Cerda J, Nolin TD, Askenazi D, Mour G, Harrell FE Jr, Pullen N, et al. ASN AKI Advisory Group (2015) Bridging translation by improving preclinical study design in AKI. J Am Soc Nephrol 26:2905-2916.

Do B, Mace M, and Rexwinkle A (2016) Idelalisib for treatment of B-cell malignancies. Am J Health Syst Pharm 73:547-555.

Doukas J, Eide L, Stebbins K, Racanelli-Layton A, Dellamary L, Martin M, Dneprovskaia E, Noronha G, Soll R, Wrasidlo W, et al. (2009) Aerosolized phosphoinositide 3-kinase gamma/delta inhibitor TG100-115 [3-[2,4-diamino-6-(3-hydroxyphenyl)pteridin-7-yl]phenol] as a therapeutic candidate for asthma and chronic obstructive pulmonary disease. J Pharmacol Exp Ther 328:758-765.

Douwes J, Gibson P, Pekkanen J, and Pearce N (2002) Non-eosinophilic asthma: importance and possible mechanisms. Thorax 57:643-648.

Finan PM and Thomas MJ (2004) PI 3-kinase inhibition: a therapeutic target for respiratory disease. Biochem Soc Trans 32:378-382.

Fishcer R, Tomé D, McGhee JR, and Boyaka PN (2007) Th1 and Th2 cells are required for both eosinophil- and neutrophil-associated airway inflammatory responses in mice. Biochem Biophys Res Commun 357:44-49.

Fruman DA (2004) Phosphoinositide 3-kinase and its targets in B-cell and T-cell signaling. Curr Opin Immunol 16:314-320.

Ghigo A, Damilano F, Braccini L, and Hirsch E (2010) PI3K inhibition in inflammation: toward tailored therapies for specific diseases. BioEssays 32:185-196.

Gidyk DC, Deibel SH, Hong NS, and McDonald RJ (2015) Barriers to developing a valid rodent model of Alzheimer's disease: from behavioral analysis to etiological mechanisms. Front Neurosci 9:245.

Gil MA, Caniga M, Woodhouse JD, Eckman J, Lee HH, Salmon M, Naber J, Hamilton VT, Sevilla RS, Bettano K, et al. (2014) Anti-inflammatory actions of chemoattractant receptor-homologous molecule expressed on Th2 by the antagonist MK-7246 in a novel rat model of Alternaria alternata elicited pulmonary inflammation. Eur J Pharmacol 743:106-116.

Grainge C, Thomas PS, Mak JC, Benton MJ, Lim TK, and Ko FW (2016) Year in review 2015: asthma and chronic obstructive pulmonary disease. Respirology 21: $765-775$

Han MK, Martinez CH, Au DH, Bourbeau J, Boyd CM, Branson R, Criner GJ, Kalhan R, Kallstrom TJ, King A, et al. (2016) Meeting the challenge of COPD care delivery in the USA: a multiprovider perspective. Lancet Respir Med 4:473-526.

Hawkins PT and Stephens LR (2015) PI3K signalling in inflammation. Biochim Biophys Acta 1851:882-897.

Hisada T, Adcock IM, Nasuhara Y, Salmon M, Huang TJ, Barnes PJ, and Chung KF (1999) Inhibition of ozone-induced lung neutrophilia and nuclear factor-kappaB binding activity by vitamin A in rat. Eur J Pharmacol 377:63-68.

Horak F, Puri KD, Steiner BH, Holes L, Xing G, Zieglmayer P, Zieglmayer R, Lemell $\mathrm{P}$, and Yu A (2016) Randomized phase 1 study of the phosphatidylinositol 3-kinase $\delta$ inhibitor idelalisib in patients with allergic rhinitis. $J$ Allergy Clin Immunol 137: 1733-1741.

Horiguchi M, Oiso Y, Sakai H, Motomura T, and Yamashita C (2015) Pulmonary administration of phosphoinositide 3-kinase inhibitor is a curative treatment for chronic obstructive pulmonary disease by alveolar regeneration. J Control Release 213:112-119.

Hsu AC, Starkey MR, Hanish I, Parsons K, Haw TJ, Howland LJ, Barr I, Mahony JB, Foster PS, Knight DA, et al. (2015) Targeting PI3K-p110 $\alpha$ suppresses influenza virus infection in chronic obstructive pulmonary disease. Am J Respir Crit Care Med 191:1012-1023.

Hylkema MN, Hoekstra MO, Luinge M, and Timens W (2002) The strength of the OVA-induced airway inflammation in rats is strain dependent. Clin Exp Immunol 129:390-396.

Kral JB, Kuttke M, Schrottmaier WC, Birnecker B, Warszawska J, Wernig C, Paar H, Salzmann M, Sahin E, Brunner JS, et al. (2016) Sustained PI3K activation exacerbates BLM-induced lung fibrosis via activation of pro-inflammatory and pro-fibrotic pathways [published correction appears in Sci Rep (2016) 7:26048]. Sci Rep 6:23034. Lee KS, Jeong JS, Kim SR, Cho SH, Kolliputi N, Ko YH, Lee KB, Park SC, Park HJ, and Lee YC (2016) Phosphoinositide 3-kinase- $\delta$ regulates fungus-induced allergic lung inflammation through endoplasmic reticulum stress. Thorax 71:52-63.

Ma C, Zhu L, Wang J, He H, Chang X, Gao J, Shumin W, and Yan T (2015) Antiinflammatory effects of water extract of Taraxacum mongolicum hand.-Mazz on lipopolysaccharide-induced inflammation in acute lung injury by suppressing PI3K/Akt/mTOR signaling pathway. J Ethnopharmacol 168:349-355.

Marwick JA, Chung KF, and Adcock IM (2010) Phosphatidylinositol 3-kinase isoforms as targets in respiratory disease. Ther Adv Respir Dis 4:19-34.

McGonigle P and Ruggeri B (2014) Animal models of human disease: challenges in enabling translation. Biochem Pharmacol 87:162-171.

McNamee K, Williams R, and Seed M (2015) Animal models of rheumatoid arthritis: how informative are they? Eur J Pharmacol 759:278-286.

Moy LY, Jia Y, Caniga M, Lieber G, Gil M, Fernandez X, Sirkowski E, Miller R, Alexander JP, Lee HH, et al. (2013) Inhibition of spleen tyrosine kinase attenuates allergen-mediated airway constriction. Am J Respir Cell Mol Biol 49:1085-1092.

Perry MWD, Abdulai R, Mogemark M, Petersen J, Thomas MJ, Valastro B, and Westin Eriksson A (2018) Evolution of PI3K $\gamma$ and $\delta$ inhibitors for inflammatory and autoimmune diseases. J Med Chem DOI: 10.1021/acs.jmedchem.8b01298 [published ahead of print].

Powell CV (2016) Acute severe asthma. J Paediatr Child Health 52:187-191.

Ramalho R, Soares R, Couto N, and Moreira A (2011) Tachykinin receptors antagonism for asthma: a systematic review. BMC Pulm Med 11:41.

Romagnani S (2002) Cytokines and chemoattractants in allergic inflammation. Mol Immunol 38:881-885.

Sanford DS, Wierda WG, Burger JA, Keating MJ, and O’Brien SM (2015) Three newly approved drugs for chronic lymphocytic leukemia: incorporating ibrutinib, 
idelalisib, and obinutuzumab into clinical practice. Clin Lymphoma Myeloma Leuk 15:385-391.

Singh PM, Borle A, Rewari V, Makkar JK, Trikha A, Sinha AC, and Goudra B (2016) Aprepitant for postoperative nausea and vomiting: a systematic review and metaanalysis. Postgrad Med J 92:87-98.

Stark AK, Sriskantharajah S, Hessel EM, and Okkenhaug K (2015) PI3K inhibitors in inflammation, autoimmunity and cancer. Curr Opin Pharmacol 23:82-91.

Takeda M, Ito W, Tanabe M, Ueki S, Kihara J, Kato H, Tanigai T, Kayaba H, Sasaki $\mathrm{T}$, and Chihara J (2010) The pathophysiological roles of PI3Ks and therapeutic potential of selective inhibitors in allergic inflammation. Int Arch Allergy Immunol 152 (Suppl 1):90-95.

Tsukagoshi H, Haddad EB, Sun J, Barnes PJ, and Chung KF (1995) Ozone-induced airway hyperresponsiveness: role of superoxide anions, NEP, and BK receptors. $J$ Appl Physiol (1985) 78:1015-1022.

Vanfleteren LEGW, Spruit MA, Wouters EFM, and Franssen FME (2016) Management of chronic obstructive pulmonary disease beyond the lungs. Lancet Respir Med 4:911-924.

Wendler A and Wehling M (2010) The translatability of animal models for clinical development: biomarkers and disease models. Curr Opin Pharmacol 10:601-606.
Wenzel SE, Schwartz LB, Langmack EL, Halliday JL, Trudeau JB, Gibbs RL, and Chu HW (1999) Evidence that severe asthma can be divided pathologically into two inflammatory subtypes with distinct physiologic and clinical characteristics. Am J Respir Crit Care Med 160:1001-1008.

Yan Z, Kui Z, and Ping Z (2014) Reviews and prospectives of signaling pathway analysis in idiopathic pulmonary fibrosis. Autoimmun Rev 13:1020-1025.

Zhao P, Zhou WC, Li DL, Mo XT, Xu L, Li LC, Cui WH, and Gao J (2015) Total glucosides of Danggui Buxue Tang attenuate BLM-induced pulmonary fibrosis via regulating oxidative stress by inhibiting NOX4. Oxid Med Cell Longev 2015: 645814.

Zhou W, Mo X, Cui W, Zhang Z, Li D, Li L, Xu L, Yao H, and Gao J (2016) Nrf? inhibits epithelial-mesenchymal transition by suppressing snail expression during pulmonary fibrosis. Sci Rep 6:38646.

Address correspondence to: Dr. Robbie L. McLeod, In Vivo Pharmacology Respiratory and Immunology, Merck \& Co., BMB 10-112, 33 Avenue Louis Pasteur, Boston, MA 02115. E-mail: robbie.mcleod@merck.com 